\title{
Fast image super-resolution algorithm based on multi-resolution dictionary learning and sparse representation
}

\author{
ZHAO Weil ${ }^{1, *}$, BIAN Xiaofeng ${ }^{1}$, HUANG Fang $^{1}$, WANG Jun ${ }^{1}$, and ABIDI Mongi A. ${ }^{2}$ \\ 1. School of Electronics and Information Engineering, Beihang University, Beijing 100191, China; \\ 2. Department of Electrical Engineering and Computer Science, University of Tennessee, Knoxville TN 37996, USA
}

\begin{abstract}
Sparse representation has attracted extensive attention and performed well on image super-resolution (SR) in the last decade. However, many current image SR methods face the contradiction of detail recovery and artifact suppression. We propose a multi-resolution dictionary learning (MRDL) model to solve this contradiction, and give a fast single image SR method based on the MRDL model. To obtain the MRDL model, we first extract multi-scale patches by using our proposed adaptive patch partition method (APPM). The APPM divides images into patches of different sizes according to their detail richness. Then, the multiresolution dictionary pairs, which contain structural primitives of various resolutions, can be trained from these multi-scale patches. Owing to the MRDL strategy, our SR algorithm not only recovers details well, with less jag and noise, but also significantly improves the computational efficiency. Experimental results validate that our algorithm performs better than other SR methods in evaluation metrics and visual perception.
\end{abstract}

Keywords: single image super-resolution (SR), sparse representation, multi-resolution dictionary learning (MRDL), adaptive patch partition method (APPM).

DOI: $10.21629 /$ JSEE.2018.03.04

\section{Introduction}

High-quality images, containing high density pixels and more detail information, are desired in various fields, such as medical diagnosis, satellite imaging and computer vision. However, images acquired by camera are mostly degraded by blur, down-sample and noise because of the limitation of imaging technology and camera chip. The degraded process can be generally modeled as a linear relationship:

$$
\boldsymbol{Y}=\boldsymbol{A X}+\boldsymbol{\eta}
$$

where $\boldsymbol{A}$ represents blurring and down-sampling operators and the random noise $\boldsymbol{\eta}$ is added. Image super-resolution (SR) $[1-15]$ is a technology that recovers a highresolution (HR) image $\boldsymbol{X}$ from the low-resolution (LR)

Manuscript received January 24, 2017.

*Corresponding author. images $\boldsymbol{Y}$. For image SR, not only the image quality is improved, but also the image resolution is also increased.

There are three major categories of image SR methods: interpolation-based methods [12-15], reconstructionbased methods [3,8,11] and learning-based methods $[1,2,4-7,9,10,16-20]$. In the early development stage of image SR, interpolation-based methods have been deeply studied, from the simple bi-cubic interpolation [15] and non-uniform interpolation [14] to the edge guided interpolators $[12,13]$, which map pixels of LR images into the HR image grid through the weighted average of neighborhood pixels. Interpolation-based methods are of high computational efficiency and easy to implement. However, the priori knowledge used in interpolation-based methods is relatively limited, and extra useful high frequency information is not brought in. Hence, the reconstructed HR images by interpolation-based methods are always fuzzy and easily affected by noise.

In order to make better use of prior information, multiple LR images are employed in reconstruction-based methods. The complementary information among multiple LR images, as prior information, is exploited to reconstruct the HR image. Villena et al. [3] combined sparse and non-sparse priors to recover the HR image. He et al. [11] modified the cost function to preserve the sharpness of edges and alleviate the outliers' effect. Babacan et al. [8] focused on estimating the HR image and motion parameters simultaneously. Even though the complementary information among LR images is very useful for image SR, the registration error and the insufficient number of LR images would result in bad restored effects by reconstructionbased methods.

Since it could be used to solve the aforementioned shortcomings of reconstruction-based methods, the learningbased method, also known as example-learning-based method, has received considerable attention in recent years. Because only a single LR image is required to recon- 
struct the HR image, image registration is not taken into account at all. The extra information or prior is provided by the learning model, which has been trained on external database offline. Among learning-based methods, sparse representation theory is very popular. In [9,10], Yang et al. presented the sparse coding for image SR (ScSR) to train HR and LR dictionary pair jointly, and assumed LR and HR image patches share the same sparse representation. Different from Yang et al.'s method, a linear relationship between two sparse coefficients was supposed by Wang et al. [6], and the dictionary pair and a mapping function were simultaneously learned. He et al. [4] presented a beta process model for the sparse representation, which contributed to generating a consistent and accurate mapping between coupled feature spaces.

Nevertheless, most of the learning-based methods face the conflict between clearness and artifacts for the reconstructed image. The ScSR method [9] can well recover image edges but generate ringing artifacts in the estimated HR image. The ringing artifacts can be alleviated by parameter tuning, while some details are lost. Wang et al. [6] and $\mathrm{He}$ et al. [4]'s algorithms degraded the ringing artifacts to some degree but also led to ambiguity in the constructed image. In order to learn general structural primitives of natural images, image patches are always extracted from images in the preprocessing of learning-based methods. When choosing the size of the image patch, Dong et al. [7] faces the same conflict. The big patch sizes could cause ambiguity in image edges, while small patch sizes tend to generate some artifacts in the reconstructed image. Therefore, adopting different patch sizes for different areas can deal with the conflict between clearness and artifacts. Based on this idea, Fu et al. [2] proposed a patchwise method for single image SR. However, their dictionaries for different regions were still trained by unclassified training patches, the reconstructed results were not improved very much. Similarly, Yeganli et al. [20] presented the patch sharpness measure (SM) defined via the gradient operator for single image SR to train specific coupled dictionary pair with respect to the different regions. However, their multiple learned dictionaries do not contain the multi-scale information.

Meanwhile, computational efficiency is another factor that influences the application of learning-based methods. Because of large training patches, high-dimensional dictionaries and iterative learning algorithms, the learningbased methods are always time-consuming. Generally, algorithms are speeded up by simplifying the learning model. Zeyde et al. [5] simplified Yang et al.'s algorithm by presenting HR dictionary through a pseudo-inverse expression, which obtained similar reconstruction results with less running time. In [1], a magnified LR input image, denoted as the pseudo-HR image, was employed in training the only HR dictionary without an additional HR images training set. The learning model was greatly simplified and the total time was reduced, but the reconstruction results were not very satisfactory.

In this paper, we propose a fast SR method based on sparse representation to solve the contradiction of improving edge clearness and getting rid of artifacts in the single image SR process. The idea of our SR algorithm is to build a multi-resolution dictionary learning (MRDL) model. To get the MRDL model, training images are firstly partitioned into multi-scale patches by the adaptive patch partition method (APPM). According to richness of image details, the APPM employs different patch sizes for different areas. The largest patches are used for flat areas, and the smallest patches for detail-richest areas. After that, the multi-resolution dictionaries can be generated by training these multi-scale patches via sparse coding algorithm. Different from the single resolution dictionary only suitable for special areas [9], the novel dictionaries contain structural primitives of various resolutions and are more general for most natural images. During image reconstruction, the input LR image is also partitioned into different size patches according to their detail richness, and reconstructed based on the dictionary of corresponding resolution. Hence, our algorithm not only performs very well on recovering image edges, but also can restrain visual artifacts. Furthermore, the MRDL strategy greatly speeds up the training and reconstruction processes. This is because the APPM enormously reduces the number of patches by employing a large patch size for flat areas. Additionally, the MRDL model lessens dictionary atoms as much as possible on the condition of ensuring the dictionary completeness.

The remainder of this paper is organized as follows: Section 2 introduces the MRDL model and details the APPM. In Section 3, we elaborate the entire image reconstruction process and present the framework of our algorithm. Various experimental results demonstrated in Section 4 evaluate the effect of our method on image SR, and some parameter settings are also analyzed. A final conclusion is made in Section 5.

\section{Multi-resolution dictionary learning model for single image $S R$}

The sparse-representation-based image SR methods are always involved in selecting image patches from a large database and then training dictionary from these patches. The image patch size selection has a great influence on dictionary. Dictionary trained by large patches contains ob- 
ject structures, while small patches chiefly reflect the detailed changes of pixel values. To reconstruct the high resolution image perfectly, it is necessary to construct multiresolution dictionaries, the atoms of which are trained by patches of different sizes. However, most of the image SR methods based on sparse representation only include single resolution dictionary $[1,5-7,9,10,21]$, which is trained by the single size image patches. In the following, we will present our MRDL model, however, the image patch partition method is addressed firstly.

\subsection{APPM}

For most natural images, they simultaneously have flat areas and detail-rich areas whose details are at different levels. In this part, we partition the original images into patches of different sizes according to their detail richness.

In order to focus the dictionary training on characterizing image edges and textures, HR images $\boldsymbol{X}^{\text {train }}=$ $\left\{\boldsymbol{X}_{j}^{\text {train }}\right\}_{j}$ and LR images $\boldsymbol{Y}^{\text {train }}=\left\{\boldsymbol{Y}_{j}^{\text {train }}\right\}_{j}$ are firstly pre-processed by high-pass filtering respectively. LR image features $f_{Y}$ are extracted by feature extraction operators $F$, and differences between HR and LR images are regarded as HR image features $f_{X}$. For LR images, we set $n$ kinds of patch sizes which are referred as $\left\{N_{1} \times\right.$ $\left.N_{1}, N_{2} \times N_{2}, \ldots, N_{n} \times N_{n}\right\}$ where $N_{1}>N_{2}>\cdots>N_{n}$. For the corresponding HR images, patch sizes are set as $\left\{s N_{1} \times s N_{1}, s N_{2} \times s N_{2}, \ldots, s N_{n} \times s N_{n}\right\}$, where $s$ is a scale factor. At first, the LR images are up-sampled to the same size as that of HR images by bi-cubic interpolation. Then, we partition the magnified LR images into patches with size $s N_{1} \times s N_{1}$ and calculate the variance of each patch. If the variance of one patch is bigger than partition threshold $\sigma_{1}$, which is determined by K-means clustering algorithm $[6,22,23]$, we will continually partition this patch into patches of size $s N_{2} \times s N_{2}$. Otherwise, the LR and HR feature patches will be unchanged and assigned to the first category. With respect to the selection of partition threshold $\sigma_{1}$, more specifically we first use K-means to split the patches into two clusters according to the variance of each patch. In the cluster which contains lower variance patches, we then choose the max variance of the patches as the value of partition threshold $\sigma_{1}$. This operation will be repeated until the patch size reaches the minimum value $s N_{n}$. Eventually, we can obtain $n$ categories of LR feature patches $\boldsymbol{P}_{l}^{\text {train }}=\left\{\boldsymbol{P}_{l 1}^{\text {train }}, \boldsymbol{P}_{l 2}^{\text {train }}, \ldots, \boldsymbol{P}_{l n}^{\text {train }}\right\}$ and HR feature patches $\boldsymbol{P}_{h}^{\text {train }}=\left\{\boldsymbol{P}_{h 1}^{\text {train }}, \boldsymbol{P}_{h 2}^{\text {train }}, \ldots, \boldsymbol{P}_{h n}^{\text {train }}\right\}$.

The $n$ categories of feature patches, generated by APPM, can exactly represent multi-level LR image features and extra HR information. This can be visually verified by the experiment in Fig. 1. In this experiment, we first reconstruct the HR image "Monarch" using three kinds of patch sizes $8 \times 8,4 \times 4$ and $2 \times 2$ respectively, and then segment the three recovered HR images into $9 \times 9$ patches. RMSEs of corresponding patches of these three images are compared. In Fig. 1(b), green color denotes that RMSEs of these areas are the highest when patch size $2 \times 2$ is used to reconstruct the HR image. Blue color indicates these areas are more suitable to be recovered with patch size $4 \times 4$. Using patch size $8 \times 8$ can obtain the higher RMSEs in red color areas. The conclusion can be made from Fig. 1(b) that small patch $2 \times 2$ is good at reconstructing detail-rich areas and large patch $8 \times 8$ is suitable for flat areas. In Fig. 1(c), we set $n=3, s=3$ and $N_{1}=8, N_{2}=4, N_{3}=$ 2 , and partition the image using our APPM. The green, blue, red colors in Fig. 1(c) respectively denotes $2 \times 2$, $4 \times 4,8 \times 8$ patches generated by APPM. Comparing Fig. 1(b) and Fig. 1(c), we can see that distributions of different size patches obtained by APPM generally conform to the optimal RMSEs experiment.

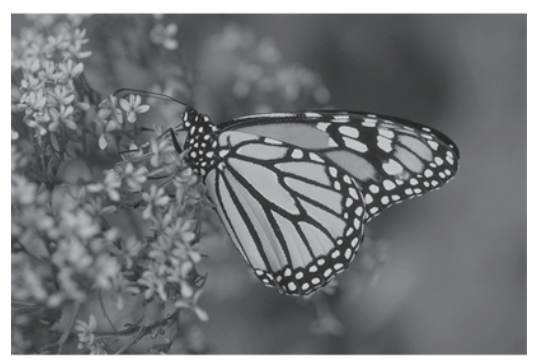

(a) The original image

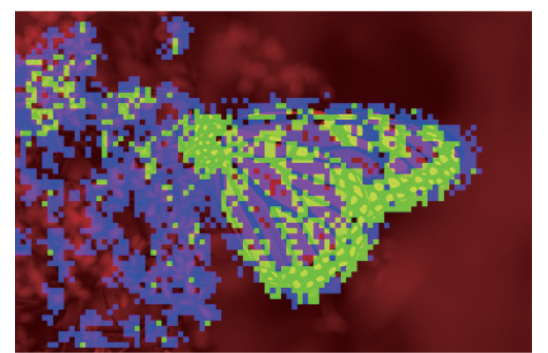

(b) Patch-size distributions by the optimal RMSEs experiment

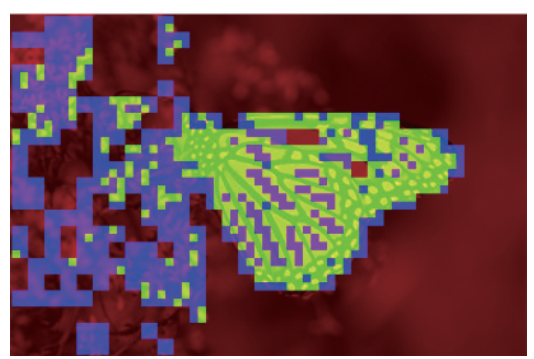

(c) Partition result by the APPM

Fig. 1 Effect of APPM

\subsection{Learning multi-resolution dictionary pairs}

In this section, a new MRDL model is proposed to learn 
the accurate mapping between LR and HR images in sparse domains. Unlike the existing dictionary learning models $[1,4-7,9,10,21]$, our learning model adopts classified image features to train dictionaries, and each category feature patch has its own special size.

After patch partition, the multi-resolution LR dictionaries $\left\{\boldsymbol{\Phi}_{l i}\right\}_{i=1,2, \ldots, n}$ and HR dictionaries $\left\{\boldsymbol{\Phi}_{h i}\right\}_{i=1,2, \ldots, n}$ as well as sparse coefficients $\left\{\boldsymbol{\alpha}_{l i}, \boldsymbol{\alpha}_{h i}\right\}_{i=1,2, \ldots, n}$ can be trained from $n$ categories of LR feature patches $\boldsymbol{P}_{l}^{\text {train }}=\left\{\boldsymbol{P}_{l 1}^{\text {train }}, \boldsymbol{P}_{l 2}^{\text {train }}, \ldots, \boldsymbol{P}_{l n}^{\text {train }}\right\}$ and HR feature patches $\boldsymbol{P}_{h}^{\text {train }}=\left\{\boldsymbol{P}_{h 1}^{\text {train }}, \boldsymbol{P}_{h 2}^{\text {train }}, \ldots, \boldsymbol{P}_{h n}^{\text {train }}\right\}$. In addition, we assume sparse coefficients of HR and LR feature patches to be the same, that is $\boldsymbol{\alpha}_{l i}=\boldsymbol{\alpha}_{h i}=\boldsymbol{\alpha}_{i}$, $i=1,2, \ldots, n$. Therefore, the MRDL problem can be written as

$$
\begin{gathered}
\min _{\left\{\boldsymbol{\Phi}_{l i}, \boldsymbol{\Phi}_{h i}, \alpha_{i}\right\}}\left\|\boldsymbol{\alpha}_{i}\right\|_{1}, \quad i=1,2, \ldots, n \\
\text { s.t. }\left\|\boldsymbol{P}_{l i}^{\text {train }}-\boldsymbol{\Phi}_{l i} \cdot \boldsymbol{\alpha}_{i}\right\|_{2}^{2} \leqslant \varepsilon_{1} \\
\left\|\boldsymbol{P}_{h i}^{\text {train }}-\boldsymbol{\Phi}_{h i} \cdot \boldsymbol{\alpha}_{i}\right\|_{2}^{2} \leqslant \varepsilon_{2} .
\end{gathered}
$$

Recently, the Lagrange Dual method [7,9,10] is predominantly used to solve this optimization problem:

$$
\begin{gathered}
\min _{\left\{\boldsymbol{\Phi}_{l i}, \boldsymbol{\Phi}_{h i}, \boldsymbol{\alpha}_{i}\right\}} \frac{1}{M_{i}}\left\|\boldsymbol{P}_{l i}^{\text {train }}-\boldsymbol{\Phi}_{l i} \cdot \boldsymbol{\alpha}_{i}\right\|_{2}^{2}+ \\
\frac{1}{N_{i}}\left\|\boldsymbol{P}_{h i}^{\text {train }}-\boldsymbol{\Phi}_{h i} \cdot \boldsymbol{\alpha}_{i}\right\|_{2}^{2}+\lambda\left(\frac{1}{M_{i}}+\frac{1}{N_{i}}\right)\left\|\boldsymbol{\alpha}_{i}\right\|_{1} \\
i=1,2, \ldots, n
\end{gathered}
$$

where $M_{i}$ and $N_{i}$ are dimensions of the $i$ th category LR and HR image feature patches in the vector form, respectively. Here, $1 / M_{i}$ and $1 / N_{i}$ are to balance the three terms of (3). To make further approximation, the constrained optimization (3) can be compactly reformulated as

$$
\begin{gathered}
\min _{\left\{\boldsymbol{\Phi}_{i}, \boldsymbol{\alpha}_{i}\right\}}\left\|\boldsymbol{P}_{i}^{\text {train }}-\boldsymbol{\Phi}_{i} \cdot \boldsymbol{\alpha}_{i}\right\|_{2}^{2}+\widetilde{\lambda}\left\|\boldsymbol{\alpha}_{i}\right\|_{1}, \\
i=1,2, \ldots, n \\
\text { where } \boldsymbol{P}_{i}^{\text {train }}=\left[\begin{array}{l}
\frac{1}{\sqrt{N_{i}}} \boldsymbol{P}_{h i}^{\text {train }} \\
\frac{1}{\sqrt{M_{i}}} \boldsymbol{P}_{l i}^{\text {train }}
\end{array}\right], \boldsymbol{\Phi}_{i}=\left[\begin{array}{l}
\frac{1}{\sqrt{N_{i}}} \boldsymbol{\Phi}_{h i} \\
\frac{1}{\sqrt{M_{i}}} \boldsymbol{\Phi}_{l i}
\end{array}\right],
\end{gathered}
$$
and $\widetilde{\lambda}=\lambda\left(\frac{1}{M_{i}}+\frac{1}{N_{i}}\right)$. Notice that this is a linear regression regularized with $\ell_{1}$-norm on the coefficients, known as the lasso problem. In this paper, we adopt the efficient sparse coding algorithm proposed in [24] to learn the overcomplete dictionary. The whole procedure of MRDL is summarized by Algorithm 1.

Algorithm 1 MRDL Step
Input: The training HR images $\boldsymbol{X}^{\text {train }}=\left\{\boldsymbol{X}_{j}^{\text {train }}\right\}_{j}$, LR images $\boldsymbol{Y}^{\text {train }}=\left\{\boldsymbol{Y}_{j}^{\text {train }}\right\}_{j}$, and the multi-resolution dictionary sizes $k_{i=1,2, \ldots, n}$.

Initialize: Upscale $\boldsymbol{Y}^{\text {train }}$ to magnified images $\widehat{\boldsymbol{Y}}^{\text {train }}$ by a scale factor $s$ and extract gradient features $f_{\boldsymbol{Y}}$ from $\widehat{\boldsymbol{Y}}^{\text {train }}$. Remove the low-frequencies from $\boldsymbol{X}^{\text {train }}: f_{\boldsymbol{X}}=$ $\boldsymbol{X}^{\text {train }}-\widehat{\boldsymbol{Y}}^{\text {train }}$.

Output: Multi-scale LR and HR feature patches $\boldsymbol{P}_{l}^{\text {train }}=\left\{\boldsymbol{P}_{l 1}^{\text {train }}, \boldsymbol{P}_{l 2}^{\text {train }}, \ldots, \boldsymbol{P}_{l n}^{\text {train }}\right\}, \quad \boldsymbol{P}_{h}^{\text {train }}=$ $\left\{\boldsymbol{P}_{h 1}^{\text {train }}, \boldsymbol{P}_{h 2}^{\text {train }}, \ldots, \boldsymbol{P}_{h n}^{\text {train }}\right\}$. The multi-resolution dictionary pairs $\left\{\boldsymbol{\Phi}_{h i}\right\}_{i=1,2, \ldots, n}$ and $\left\{\boldsymbol{\Phi}_{l i}\right\}_{i=1,2, \ldots, n}$. The PCA projection operator $\left\{\boldsymbol{B}_{i}\right\}_{i=1,2, \ldots, n}$.

Procedure: Multi-scale training feature patches

Step 1 Calculate thresholds $\boldsymbol{\sigma}=\left[\sigma_{1}, \sigma_{2}, \ldots, \sigma_{n-1}\right]$ automatically by the K-means clustering algorithm.

Step 2 For extract $s N_{1} \times s N_{1}$ patch $p_{1 m}$ from images $\widehat{\boldsymbol{Y}}^{\text {train }}$, and calculate the variance $v_{1 m}$ of $p_{1 m}$.

Step 3 If $v_{1 m}<\sigma_{1}$

Extract LR feature patch $p_{1 m}^{\boldsymbol{Y}}$ from $f_{\boldsymbol{Y}}$ and put it to $P_{l 1}^{\text {train }}$.

Extract HR feature patch $p_{1 m}^{\boldsymbol{X}}$ from $f_{\boldsymbol{X}}$ and put it to $P_{h 1}^{\text {train }}$.

Step 4 Else divide $p_{1 m}$ into $s N_{2} \times s N_{2}$ patches.

Step 5 For each $s N_{2} \times s N_{2}$ patch $p_{2 u}$, calculate its variance $v_{2 u}$.

Step 6 If $v_{2 u}<\sigma_{2}$

Extract LR feature patch $p_{2 u}^{\boldsymbol{Y}}$ from $f_{\boldsymbol{Y}}$ and put it to $P_{l 2}^{\text {train }}$.

Extract HR feature patch $p_{2 u}^{\boldsymbol{X}}$ from $f_{\boldsymbol{X}}$ and put it to $P_{h 2}^{\text {train }}$.

Step 7 Else divide $p_{2 u}$ into $s N_{3} \times s N_{3}$ patches. (Go on $n$-time partitions until Step $3 n-1$.)

For each $s N_{n} \times s N_{n}$ patch $p_{n v}$.

Extract LR feature patch $p_{n v}^{\boldsymbol{Y}}$ from $f_{\boldsymbol{Y}}$ and put it to $P_{l n}^{\text {train }}$.

Extract HR feature patch $p_{n v}^{\boldsymbol{X}}$ from $f_{\boldsymbol{X}}$ and put it to $P_{h n}^{\text {train }}$.

\section{Multi-resolution dictionary pairs}

Step 1 Use the principal component analysis (PCA) algorithm to reduce the dimension of $\boldsymbol{P}_{l}^{\text {train }}=$ $\left\{\boldsymbol{P}_{l 1}^{\text {train }}, \boldsymbol{P}_{l 2}^{\text {train }}, \ldots, \boldsymbol{P}_{l n}^{\text {train }}\right\}$ and obtain the projection operator $\left\{\boldsymbol{B}_{i}\right\}_{i=1,2, \ldots, n}$.

Step 2 Normalize the training feature patches $\boldsymbol{P}_{l}^{\text {train }}=\left\{\boldsymbol{P}_{l 1}^{\text {train }}, \boldsymbol{P}_{l 2}^{\text {train }}, \ldots, \boldsymbol{P}_{l n}^{\text {train }}\right\}$ and $\boldsymbol{P}_{h}^{\text {train }}=$ $\left\{\boldsymbol{P}_{h 1}^{\text {train }}, \boldsymbol{P}_{h 2}^{\text {train }}, \ldots, \boldsymbol{P}_{h n}^{\text {train }}\right\}$.

Step 3 Train the multi-resolution dictionary pairs by (4):

$$
\begin{gathered}
{\left[\boldsymbol{\Phi}_{h i}, \boldsymbol{\Phi}_{l i}\right]=\text { Efficient_Sparse_Coding }\left(\left[\boldsymbol{P}_{l i}^{\text {train }},\right.\right.} \\
\left.\left.\boldsymbol{P}_{h i}^{\text {train }}\right], k_{i}\right), \quad i=1,2, \ldots, n .
\end{gathered}
$$




\section{The proposed fast SR algorithm}

Based on MRDL, a new SR algorithm is proposed with less reconstruction time and good visual perception. The flowchart of the proposed algorithm is shown in Fig. 2, which shows three parts of our algorithm, i.e. feature extraction and patch partition, dimensionality reduction and sparse representation, and image SR reconstruction.

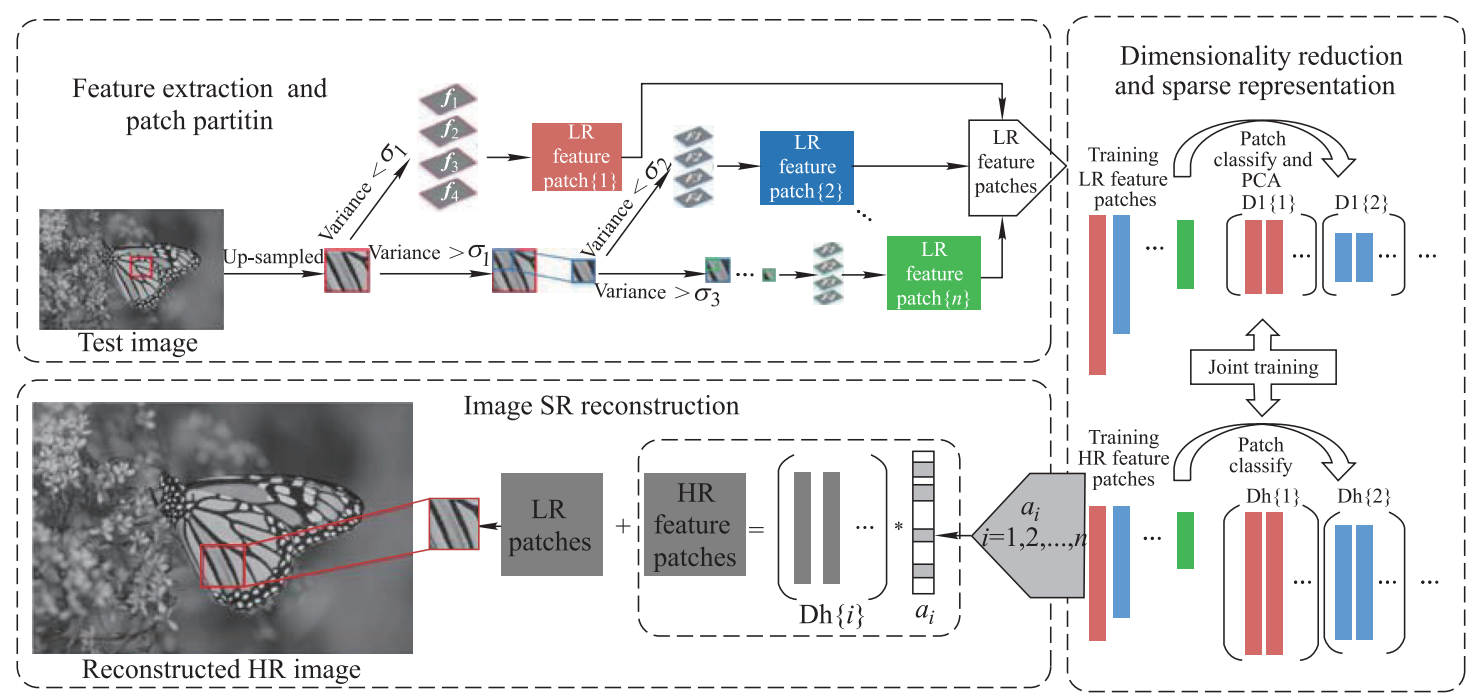

Fig. 2 Flowchart of the proposed method on image SR

\subsection{Feature extraction and patch partition}

For image SR, its main idea is to recover HR image $\boldsymbol{X}^{\text {restored }}$ by adding lost high-frequency information to LR image $\boldsymbol{Y}^{\text {test }}$. Therefore, the first and second derivatives in horizontal and vertical directions are taken as LR image features, which are similar to those employed in $[5,9,10]$ :

$$
\begin{gathered}
\boldsymbol{f}_{\boldsymbol{Y}}=\left\{\boldsymbol{f}_{1}, \boldsymbol{f}_{2}, \boldsymbol{f}_{3}, \boldsymbol{f}_{4}\right\} \otimes \widehat{\boldsymbol{Y}}^{\mathrm{test}} \\
\qquad \begin{array}{l}
\boldsymbol{f}_{1}=[-1,0,1] \\
\boldsymbol{f}_{2}=\boldsymbol{f}_{1}^{\mathrm{T}} \\
\boldsymbol{f}_{3}=[1,0,-2,0,1] \\
\boldsymbol{f}_{4}=\boldsymbol{f}_{3}^{\mathrm{T}}
\end{array}
\end{gathered}
$$

where $\mathrm{T}$ denotes transposition and $\otimes$ represents convolution. Here, we apply the four filters to the magnified image $\widehat{\boldsymbol{Y}}^{\text {test }}$ which are up-sampled by a scale factor $S$ using the bi-cubic interpolation method [15] to obtain four gradient maps.

Next, we use our APPM to partition the four gradient maps into $n$ categories of feature patches, denoted by $\boldsymbol{P}_{l}^{\text {test }}=\left\{\boldsymbol{P}_{l 1}^{\text {test }}, \boldsymbol{P}_{l 2}^{\text {test }}, \ldots, \boldsymbol{P}_{l n}^{\text {test }}\right\}$.

\subsection{Dimensionality reduction and sparse representation}

Because of the up-sampling and feature extraction, the LR image patches with size $N_{i}^{2}$, are now represented as feature patches $\boldsymbol{P}_{l i}^{\text {test }}$ of $4\left(s N_{i}\right)^{2}$ dimensions, which would result in heavy computation. In this paper, we apply the PCA algorithm [25] to reduce dimensions by ignoring $0.1 \%$ of the feature energy. Low-dimensional feature patches $\widetilde{\boldsymbol{P}}_{l}^{\text {test }}=$ $\left\{\widetilde{\boldsymbol{P}}_{l 1}^{\text {test }}, \widetilde{\boldsymbol{P}}_{l 2}^{\text {test }}, \ldots, \widetilde{\boldsymbol{P}}_{l n}^{\text {test }}\right\}$ can be obtained by multiplying $\boldsymbol{P}_{l i}^{\text {test }}$ with projection operators $\boldsymbol{B}=\left\{\boldsymbol{B}_{1}, \boldsymbol{B}_{2}, \ldots, \boldsymbol{B}_{n}\right\}$ obtained by PCA, as follows:

$$
\widetilde{\boldsymbol{P}}_{l i}^{\mathrm{test}}=\boldsymbol{B}_{i} \cdot \boldsymbol{P}_{l i}^{\mathrm{test}} .
$$

After that, the low-dimensional feature patches $\widetilde{\boldsymbol{P}}_{l}^{\text {test }}=$ $\left\{\widetilde{\boldsymbol{P}}_{l 1}^{\text {test }}, \widetilde{\boldsymbol{P}}_{l 2}^{\text {test }}, \ldots, \widetilde{\boldsymbol{P}}_{l n}^{\text {test }}\right\}$ are directly employed to acquire sparse representation $\left\{\boldsymbol{\alpha}_{i}^{*}\right\}_{i=1,2, \ldots, n}$ :

$$
\begin{gathered}
\boldsymbol{\alpha}_{i}^{*}=\arg \min _{\boldsymbol{\alpha}_{i}}\left\|\widetilde{\boldsymbol{P}}_{l i}^{\text {test }}-\boldsymbol{\Phi}_{l i} \cdot \boldsymbol{\alpha}_{i}\right\|_{2}^{2}+\lambda\left\|\boldsymbol{\alpha}_{i}\right\|_{1}, \\
i=1,2, \ldots, n
\end{gathered}
$$

where $\left\{\boldsymbol{\Phi}_{l i}\right\}_{i=1,2, \ldots, n}$ are multi-resolution LR dictionaries.

\subsection{Image SR reconstruction}

In the reconstruction stage, the sparse representation $\left\{\boldsymbol{\alpha}_{i}^{*}\right\}_{i=1,2, \ldots, n}$ and corresponding multi-resolution HR dictionaries $\left\{\boldsymbol{\Phi}_{l i}\right\}_{i=1,2, \ldots, n}$ are applied to generate HR feature patches:

$$
\widehat{\boldsymbol{P}}_{h i}^{\text {restored }}=\boldsymbol{\Phi}_{h i} \cdot \boldsymbol{\alpha}_{i}^{*}, \quad i=1,2, \ldots, n .
$$

The reconstructed HR image $\boldsymbol{X}_{0}$ can be obtained by adding LR image patches to $\widehat{\boldsymbol{P}}_{h i}^{\text {restored }}$ and stitching them into an image.

Looking back at (4), (8) and (9), the sparse representation approach just establishes the corresponding relationship between LR and HR dictionaries, and ignores the exact relationship between estimated HR image $\boldsymbol{X}_{0}$ and LR 
image $\boldsymbol{Y}^{\text {test }}$ modeled by (1). Therefore, a global reconstruction constraint is wielded to this initial HR image $\boldsymbol{X}_{0}$ as follows:

$\boldsymbol{X}^{\text {restored }}=\arg \min _{\boldsymbol{X}}\left\|\boldsymbol{D} \boldsymbol{H} \boldsymbol{X}-\boldsymbol{Y}^{\text {test }}\right\|_{2}^{2}+c\left\|\boldsymbol{X}-\boldsymbol{X}_{0}\right\|_{2}^{2}$

where $c$ is a trade-off constant. This optimization problem can be efficiently solved by the gradient descent method. The update equation for this iterative method is

$\boldsymbol{X}_{t+1}=\boldsymbol{X}_{t}+\nu\left[\boldsymbol{H}^{\mathrm{T}} \boldsymbol{D}^{\mathrm{T}}\left(\boldsymbol{Y}^{\text {test }}-\boldsymbol{D} \boldsymbol{H} \boldsymbol{X}_{t}\right)+c\left(\boldsymbol{X}-\boldsymbol{X}_{0}\right)\right]$

where $\boldsymbol{X}_{t}$ is the estimate of the HR image after the $t$ th iteration, $\nu$ is the step size of the gradient descent. The reconstructed HR image $\boldsymbol{X}^{\text {restored }}$ is regarded as the final estimated HR image in this paper.

\section{Performance evaluation}

In this section, we first demonstrate the experimental study on three important parameters of our algorithm, including patch sizes, dictionary sizes and the regularization parameter $\lambda$. After that, we present the SR results obtained by our proposed method and compare them with baseline methods.

The training dataset of our experiments is the same as that used in $[9,10]$. The training images are first downsampled by a factor $s=3$ to generate LR training images, and then patches of different size are formed to train the multi-resolution dictionary pairs. We choose twelve widely used testing images in the experiments, including cameraman and the other eleven images in Set14 [5]. The baseline methods include the bi-cubic interpolation method [15], the ScSR method [9], Fu et al.'s method [2], and two leading SR algorithms including He et al.'s method [4] and Yeganli's method [20]. All experiments are performed on a computer of Inter(R) Pentium(R) CPU G2030 @3.00 GHz with 2 GB memory. Three quality measures are used to measure the performance of four methods, that is, the peak signal-to-noise ratio (PSNR), structural similarity (SSIM) [26] and elapsed time.

\subsection{Empirical study on parameters}

The patch sizes $\left\{N_{1} \times N_{1}, N_{2} \times N_{2}, \ldots, N_{n} \times N_{n}\right\}$ of LR images, dictionary sizes $k_{i=1,2, \ldots, n}$ and the regularization parameter $\lambda$ are three sets of parameters which need to be decided in our algorithm. The size of the image patch plays a decisive role on both reconstruction quality and computational efficiency. When $n=1$, all patches have a single and same size. When $n=2$, two patch sizes are used to reconstruct HR images and so on. Besides, dictionary sizes impact the completeness of multi-resolution dictionaries, and the parameter $\lambda$ in (8) is the penalty constant for sparsity. In the following sections, we will discuss them one by one.

\subsubsection{Effects of the patch size}

In this subsection, we set $n=1,2$ and 3 respectively and discuss the proper $n$ and patch sizes through several experiments. For $n=1$, we carry out three experiments in which the patch size is set as $2 \times 2,4 \times 4$, and $8 \times 8$ respectively. The visual results are given in Fig. 3(a) - (c).

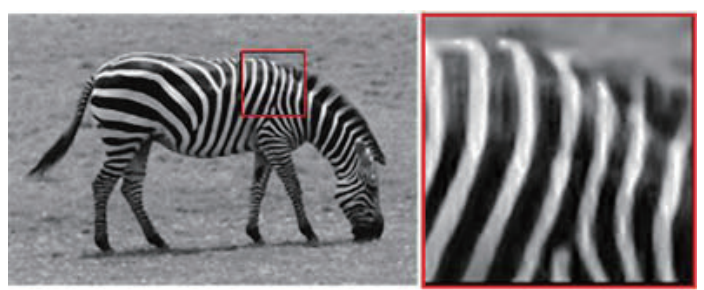

(a) LR patch size of $2 \times 2$

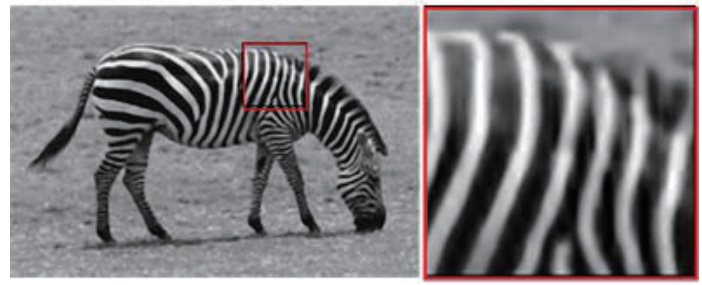

(b) LR patch size of $4 \times 4$

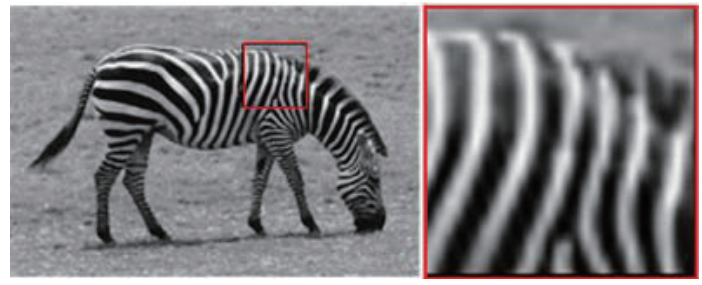

(c) LR patch size of $8 \times 8$

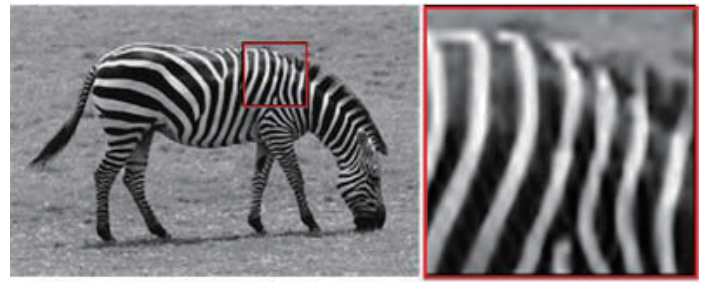

(d) LR patch size of $8 \times 8$ and $4 \times 4$

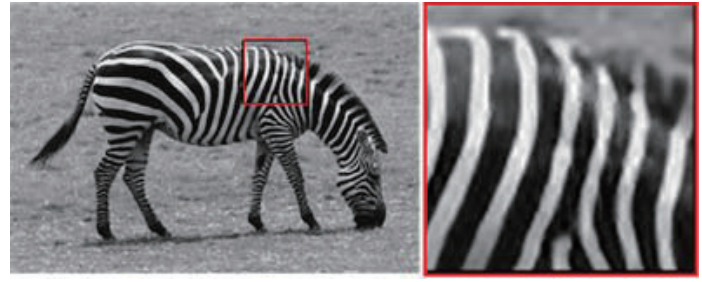

(e) LR patch size of $8 \times 8,4 \times 4$ and $2 \times 2$ 


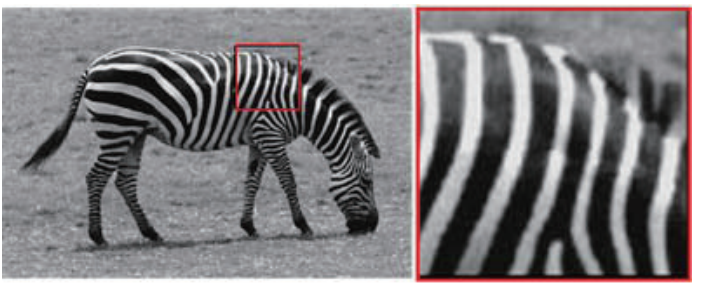

(f) The original image

Fig. 3 Visual comparison of reconstructed images by the proposed method with different patch sizes

The edges of image "Zebra" are very sharp by using $2 \times 2$ patches, but this over-sharpness leads to many artifacts generated around the edges. Using larger patches $4 \times 4$, the edges become somewhat fuzzy while many reconstruction artifacts have diminished. The first two columns of Table 1 also illustrate that using $4 \times 4$ patches to reconstruct HR images can get higher PSNRs. However, when the patch size increases to $8 \times 8$, although most of the reconstruction artifacts fade away, image edges are extremely ambiguous, thus its PSNRs are the lowest in Table 1. All dictionary sizes set to $256, \lambda=0.6$, scale factor $S=3$. From these experiments, we know that the conflict of clearness and artifacts cannot be absolutely settled by simply altering the patch size for the whole image. In addition, it can be seen from the first three columns of Table 1 that the running time is reduced by enlarging patch sizes, since large patches can decrease the number of image patches.

Table 1 PSNR and reconstruction time results by the proposed method with different patch sizes

\begin{tabular}{ccccccc}
\hline & & \multicolumn{5}{c}{ Patch size } \\
\cline { 3 - 7 } Image & Parameter & & & & $8 \times 8$ & $8 \times 8$ \\
& & & $4 \times 4$ & $8 \times 8$ & $4 \times 4$ & $4 \times 4$ \\
& & & & & $2 \times 2$ \\
\hline Barbara & PSNR/dB & 27.63 & 27.79 & 27.42 & 27.84 & 27.92 \\
$128 \times 128$ & Time/s & 0.75 & 0.38 & 0.19 & 0.35 & 0.30 \\
\hline Cameraman & PSNR/dB & 24.23 & 24.20 & 23.97 & 24.43 & 24.76 \\
$128 \times 128$ & Time/s & 0.63 & 0.34 & 0.17 & 0.29 & 0.25 \\
\hline Lena & PSNR/dB & 32.52 & 32.71 & 32.52 & 32.95 & 33.03 \\
$512 \times 512$ & Time/s & 6.08 & 2.36 & 1.28 & 2.27 & 2.12 \\
\hline Zebra & PSNR/dB & 27.69 & 27.71 & 26.77 & 28.55 & 28.58 \\
$391 \times 586$ & Time/s & 5.97 & 2.09 & 1.27 & 2.01 & 1.88 \\
\hline Monarch & PSNR/dB & 30.60 & 30.78 & 30.46 & 31.16 & 31.80 \\
$512 \times 768$ & Time/s & 9.91 & 3.50 & 1.84 & 3.10 & 2.93 \\
\hline
\end{tabular}

According to the aforementioned experiments, we know that large patches $(8 \times 8)$ can improve computational efficiency and diminish artifacts, and middle patch size $4 \times 4$ can balance the edges against artifacts. Then, for $n=2$, we first segment the whole image into $8 \times 8$ patches and then divide some $8 \times 8$ patches with more detail information into $4 \times 4$ patches. As shown in Fig. 3(d), where the edges of image "Zebra" are clearer than the result obtained by only using $8 \times 8$ patches. Nevertheless, there are still ambiguities compared with the original image. Hence we further divide some $4 \times 4$ patches with abundant edges into smaller patches $2 \times 2$. That is, for $n=3$, three kinds of patch sizes $8 \times 8,4 \times 4,2 \times 2$ are adopted in our algorithm. The reconstruction result of $n=3$ is shown in Fig. 3(e) which has the clearest edges and least artifacts among the five reconstruction images. Table 1 also demonstrates that the estimated HR images reconstructed by three kinds of patch sizes can obtain the highest PSNRs. Additionally, the running time of $n=3$ is relatively less because of most patches with large size $8 \times 8$. Thus, we set $N_{1}=8, N_{2}=4, N_{3}=2$ for our method in the following experiments.

\subsubsection{Effects of the dictionary size}

In most SR algorithms based on sparse representation $[1,2,4-6,9,10]$, the dictionary size is always set among $\{64,128,256,512,1024,2048\}$. Considering the tradeoff between reconstruction accuracy and computation efficiency, we choose the number of atoms $k_{i=1,2,3}$ among $\{128,256,512\}$ for three-resolution dictionaries whose corresponding patch sizes of LR images are $8 \times 8,4 \times 4$ and $2 \times 2$.

To explain how to choose the size for each resolution dictionary, the following experiment is carried out. The experiments are performed on 24 images in Kodak PhotoCD dataset, each of which has a mass of flat areas and detailrich areas. Every image is segmented into three kinds of sub-images with size $99 \times 99$, i.e., flat sub-images, detailricher sub-images and detail-richest sub-images. At first, all flat sub-images are reconstructed by our method with different dictionary sizes. We calculate the SSIMs of each reconstructed sub-image and show SSIM histograms in Fig. 4(a). As pointed out by [26], the SSIM value evaluates the similarity between two images. The higher SSIM is, the more similar the recovered image is to the original one. As illustrated in Fig. 4(a), SSIM distributions are almost the same for three dictionary sizes and mostly concentrates on $[0.9,1]$. It implies that 128 dictionary atoms are enough to approximately represent the flat areas. We also do the similar experiments for all detail-richer and detail-richest sub-images, the SSIM histograms are shown in Fig. 4(b) and Fig. 4(c) respectively. These experiments indicate that the number of dictionary atoms is closely related to the characteristic of input data, that is, the fewer the image edges and textures are, the feuer dictionary atoms it requires for approximate representation. 


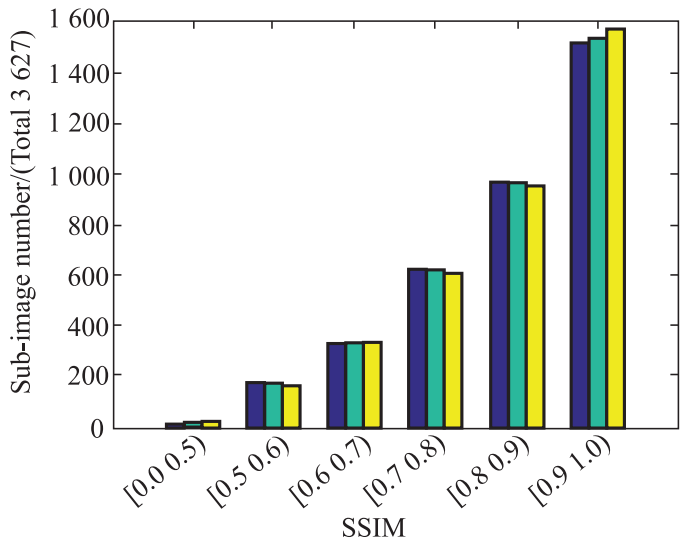

(a) Flat sub-images

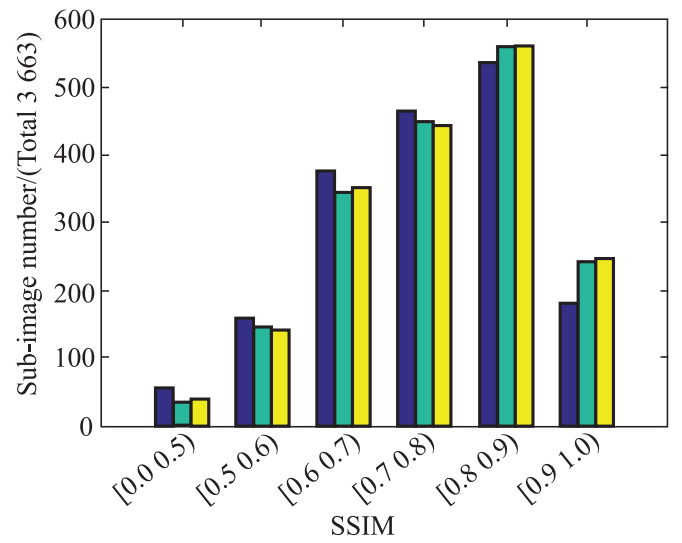

(b) Detail-richer sub-images

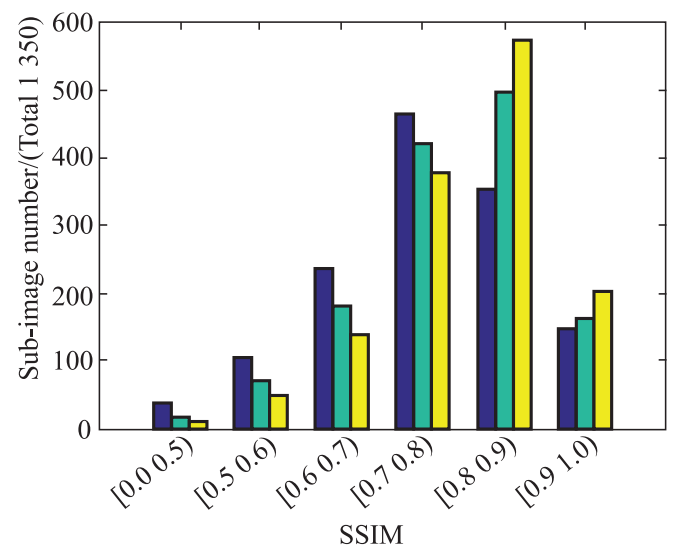

(c) Detail-richer sub-images

: 128 atoms; $\square: 256$ atoms; $\square: 512$ atoms.

Fig. 4 SSIM histograms of sub-images reconstructed by multiresolution dictionaries with different sizes

The last column of Table 2 also indicates multiresolution dictionary atoms of $[128,256,512]$ can reach higher PSNR values with shorter running time. The patch sizes set to $8 \times 8,4 \times 4,2 \times 2$ respectively, $\lambda=0.6$, scale factor $S=3$. A tradeoff between PSNRs and computation time can be achieved by setting different dictionary sizes at different resolutions.
Table 2 PSNR and reconstruction time results by the proposed method with different dictionary sizes

\begin{tabular}{cccccc}
\hline \multirow{2}{*}{ Image } & Parameter & \multicolumn{4}{c}{ Dictionary size } \\
\cline { 3 - 6 } & & 128,128, & 256,256, & 512,512, & 128,256, \\
& & 128 & 256 & 512 & 512 \\
\hline Cameraman & PSNR/dB & 31.76 & 31.91 & 32.02 & 32.00 \\
$512 \times 512$ & Time/s & 1.72 & 1.94 & 2.09 & 1.78 \\
\hline Man & PSNR/dB & 28.06 & 28.08 & 28.09 & 28.09 \\
$512 \times 512$ & Time/s & 1.84 & 1.98 & 2.13 & 1.91 \\
\hline Monarch & PSNR/dB & 31.74 & 31.80 & 31.89 & 31.87 \\
$512 \times 768$ & Time/s & 2.80 & 2.93 & 3.06 & 2.87 \\
\hline Ppt3 & PSNR/dB & 25.13 & 25.23 & 25.48 & 25.48 \\
$656 \times 529$ & Time/s & 2.53 & 2.64 & 2.87 & 2.55 \\
\hline Zebra & PSNR/dB & 28.55 & 28.57 & 28.60 & 28.58 \\
$391 \times 586$ & Time/s & 1.73 & 1.88 & 1.98 & 1.80 \\
\hline
\end{tabular}

\subsubsection{Effects of the parameter $\lambda$}

The parameter $\lambda$ in (8) has influence on the naturalness of reconstructed HR images. In this subsection, PSNR and SSIM metrics are taken to test the effect of $\lambda$ with different values, $\lambda \in[0.05,2]$, and the "Lena" image $(512 \times 512)$ is used as the test image for example.

Fig. 5 demonstrates PSNR and SSIM curves of the reconstructed "Lena" image with respect to different values of $\lambda$.

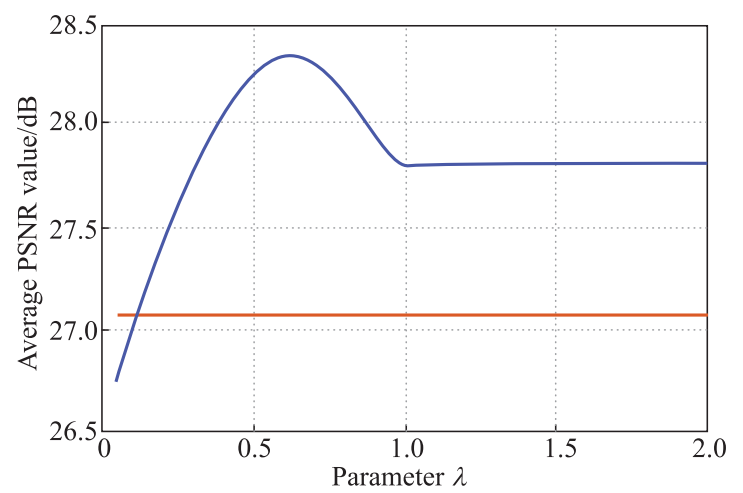

(a) PSNR

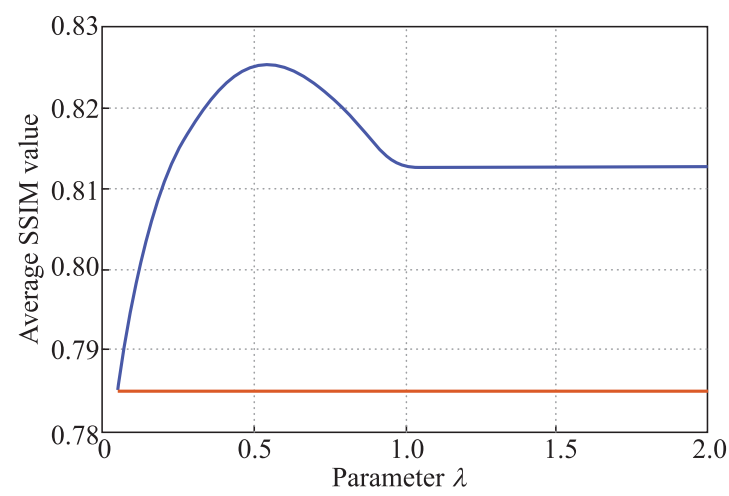

(b) SSIM

— : The proposed method; __ : Bi-cubic interpolation

Fig. 5 PSNR and SSIM curves describing the effect of parameter $\lambda$ on "Lena" image by using our proposed method 
The results show that PSNRs and SSIMs both increase rapidly as $\lambda$ varying from 0.05 to 0.6 , and then reach the maximum value near 0.6. However, as $\lambda$ continues to increase, the PSNR and SSIM values drop and become stable for $\lambda \geqslant 1$. It can also be seen from Fig. 6 that small values of $\lambda$ can enhance the edge sharpness of the "Lena" image but lead to ringing artifacts. Larger $\lambda$ can smooth the reconstructed "Lena" image to be natural but blur image details. Therefore, we choose $\lambda=0.6$ to balance the image naturalness and edge sharpness. The similar results can be obtained from other test images.

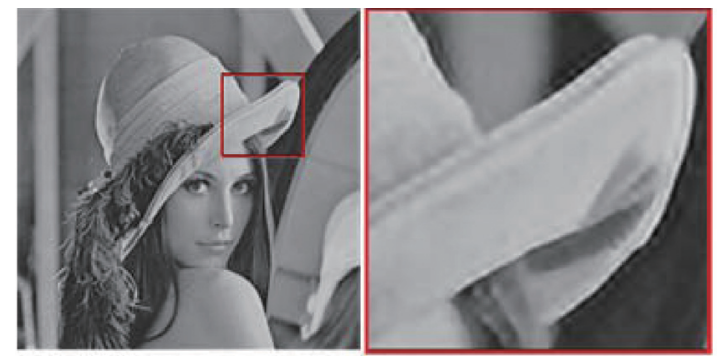

(a) $\lambda=0.4$

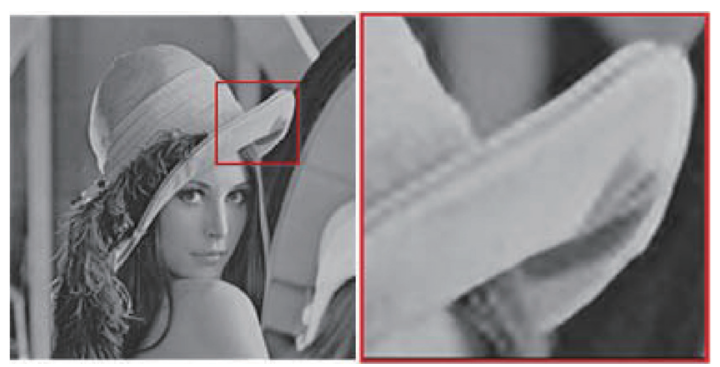

(b) $\lambda=0.6$

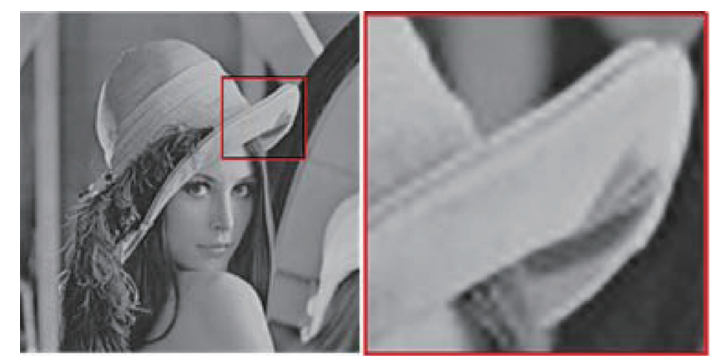

(c) $\lambda=0.8$

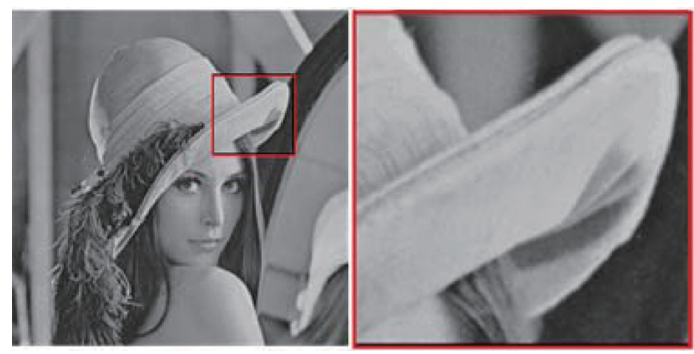

(d) The original HR image

Fig. 6 Effects of parameter $\lambda$ on the reconstructed "Lena" image

\subsection{Experimental results}

In this section, we compare our method with five other methods, i.e., the bi-cubic interpolation (BI) [15], the ScSR method [9], Fu et al.'s method [2], He et al.'s method [4] and Yeganli's method [20]. In ScSR method, 100000 LR patches of size $5 \times 5$ with 4-pixel overlaps are sampled to train the dictionary, and the sparsity regularization coefficient $\lambda$ in (8) is set as 0.2 . For Fu et al.'s method, two set of dictionaries with patch sizes $5 \times 5$ and $8 \times 8$ are trained respectively, and the test image is divided into $5 \times 5$ and $8 \times 8$ patches by a fixed threshold in the reconstruction stage. The dictionary size is fixed to 512 in the above two methods. We use the default design parameters and the training image dataset for the algorithm of $\mathrm{He}$ et al. as specified in [14], and a pair of 771-atom coupled dictionaries is trained. As for Yeganli's method, we use the same training dataset as that used in $[9,10]$. Except for this, all other algorithms' settings follow the default settings specified in [20], and the number of SM clusters is set to seven. In our method, the overlap pixels are 5, 0 and 0 respectively for LR patch sizes $8 \times 8,4 \times 4$ and $2 \times 2$. The parameter $\widetilde{\lambda}$ in (4) is empirically set as 0.15 .

The visual comparisons are showed in Fig. 7. It is noticeable that our method can achieve the best visual performance because of the MRDL model. The results of the ScSR method have many jaggy and ringing artifacts which are unwanted. Fu et al.'s method [2] can get sharper image edges while more noise residuals and artifacts are emerged in the reconstructed images. He et al. [14] employs the beta process model to replace the identical mapping assumption used in other contrast algorithms including our proposed one. Although the beta process model does greatly improve the performance, its reconstruction result is still slightly inferior to our result and highly time-consuming. It also makes sense that our proposed MRDL model is very effective. Another leading algorithm, Yeganli's method [20], uses seven coupled dictionary pairs to reconstruct the image. Each coupled dictionary pair contains different levels of edges and detail information measured by SM. Owning to lack of multi-scale information, their multiple dictionary pairs are not as effective as our multi-resolution dictionary pairs. It is worthwhile to point out that textures reconstructed by our method are much more natural, with less jag and noise. Furthermore, the image edge sharpness improves a lot. We also quantitatively evaluate the performance of these methods, and the results are showed in Table 3 . The patch sizes are $8 \times 8,4 \times 4,2 \times 2$ respectively, scale factor $S=3$. As we can see, the quantitative indexes of our method are superior to the baseline methods. Although the reconstruction time is greatly reduced 
by Fu et al.'s method [2] as well as Yeganli's method [20], our method further improves the computational efficiency, with only several seconds to obtain a reconstructed HR image.

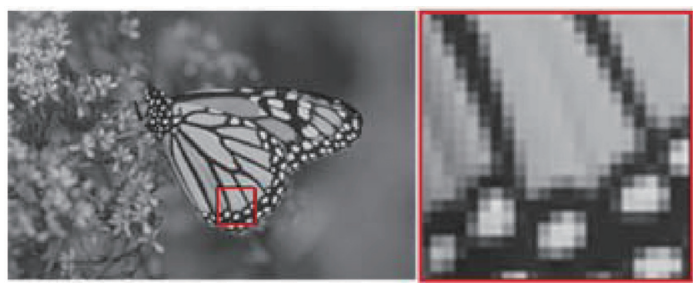

(a) Low-resolution input
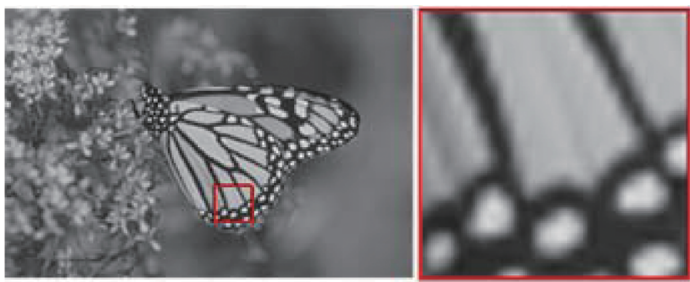

(b) BI

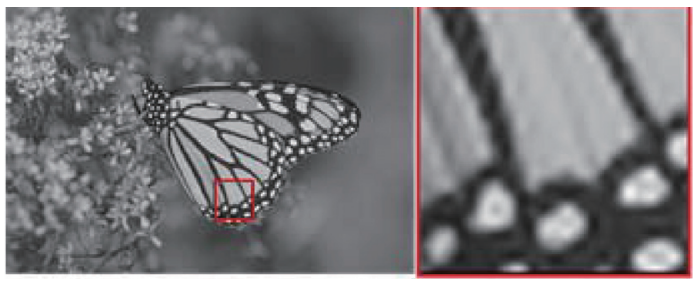

(c) Yang et al.'s method

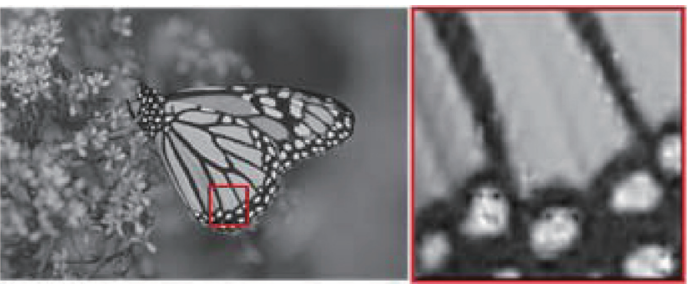

(d) Fu et al.'s method

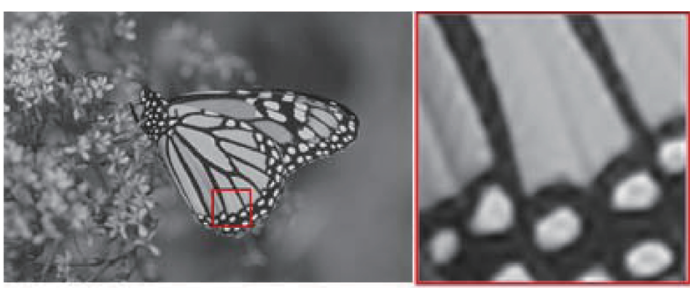

(e) He et al.'s method

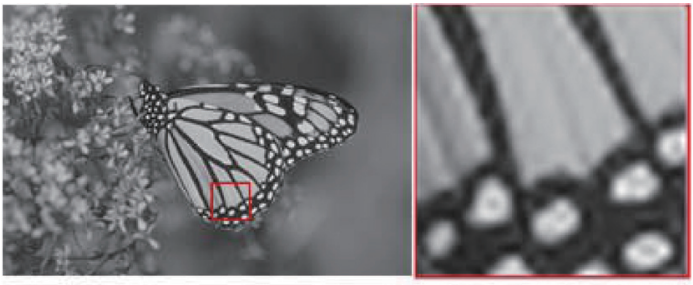

(f) Yeganli's method
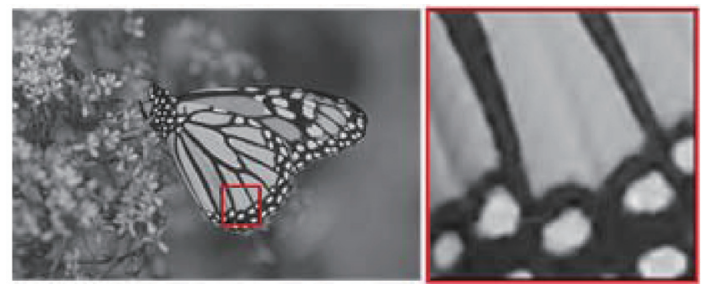

(g) Our method

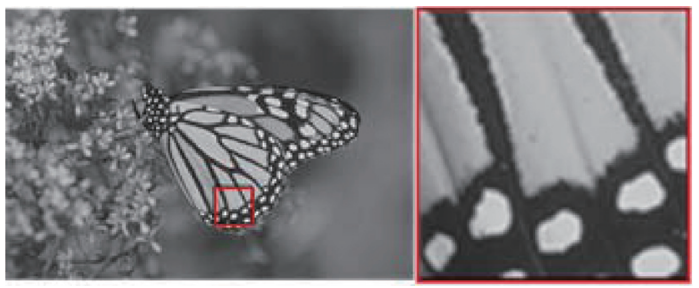

(h) The original HR image

Fig. 7 Super-resolution results for image "Monarch" of size $512 \times 768$ magnified by a factor of 3

Table 3 PSNR, SSIM and reconstruction time results for 12 test images

\begin{tabular}{|c|c|c|c|c|c|c|c|}
\hline Image & Parameter & Bicubic & ScSR & Fu et al. & He et al. & Yeganli & Our \\
\hline \multirow{3}{*}{$\begin{array}{c}\text { Barbara } \\
{[576 \times 720]}\end{array}$} & $\mathrm{PSNR} / \mathrm{dB}$ & 26.25 & 26.72 & 26.64 & 26.84 & 26.89 & 26.79 \\
\hline & SSIM & 0.753 & 0.772 & 0.777 & 0.786 & 0.789 & 0.785 \\
\hline & Time/s & 0.09 & 602.91 & 68.59 & 1157.83 & 60.66 & 3.48 \\
\hline \multirow{3}{*}{$\begin{array}{c}\text { Bridge } \\
{[512 \times 512]}\end{array}$} & $\mathrm{PSNR} / \mathrm{dB}$ & 24.40 & 24.97 & 24.81 & 25.01 & 25.07 & 25.06 \\
\hline & SSIM & 0.649 & 0.702 & 0.696 & 0.705 & 0.708 & 0.708 \\
\hline & Time/s & 0.03 & 404.84 & 44.77 & 749.18 & 38.56 & 2.08 \\
\hline \multirow{3}{*}{$\begin{array}{l}\text { Cameramar } \\
{[256 \times 256]}\end{array}$} & $\mathrm{PSNR} / \mathrm{dB}$ & 23.77 & 24.44 & 24.32 & 24.79 & 24.63 & 24.91 \\
\hline & SSIM & 0.779 & 0.802 & 0.799 & 0.816 & 0.811 & 0.821 \\
\hline & Time/s & 0.02 & 93.09 & 10.67 & 174.53 & 9.28 & 0.75 \\
\hline \multirow{3}{*}{$\begin{array}{c}\text { Flowers } \\
{[362 \times 500]}\end{array}$} & $\mathrm{PSNR} / \mathrm{dB}$ & 27.23 & 28.28 & 27.91 & 28.66 & 28.47 & 28.64 \\
\hline & SSIM & 0.801 & 0.840 & 0.827 & 0.844 & 0.840 & 0.841 \\
\hline & Time/s & 0.06 & 274.56 & 28.58 & 508.23 & 23.30 & 1.31 \\
\hline \multirow{3}{*}{$\begin{array}{c}\text { Foreman } \\
{[288 \times 352]}\end{array}$} & $\mathrm{PSNR} / \mathrm{dB}$ & 31.18 & 32.81 & 32.08 & 33.10 & 33.26 & 33.19 \\
\hline & SSIM & 0.907 & 0.924 & 0.915 & 0.928 & 0.931 & 0.930 \\
\hline & Time/s & 0.03 & 140.43 & 16.27 & 269.69 & 12.45 & 1.02 \\
\hline \multirow{3}{*}{$\begin{array}{c}\text { Lena } \\
{[256 \times 256]}\end{array}$} & $\mathrm{PSNR} / \mathrm{dB}$ & 29.94 & 30.85 & 30.75 & 31.36 & 31.30 & 31.36 \\
\hline & SSIM & 0.843 & 0.861 & 0.856 & 0.869 & 0.867 & 0.869 \\
\hline & Time/s & 0.03 & 90.17 & 9.75 & 172.76 & 7.97 & 0.69 \\
\hline \multirow{3}{*}{$\begin{array}{c}\text { Man } \\
{[512 \times 512]}\end{array}$} & PSNR/dB & 27.01 & 27.72 & 27.58 & 28.05 & 27.96 & 28.09 \\
\hline & SSIM & 0.749 & 0.784 & 0.778 & 0.791 & 0.790 & 0.792 \\
\hline & Time/s & 0.05 & 394.50 & 43.34 & 740.72 & 33.25 & 1.91 \\
\hline \multirow{3}{*}{$\begin{array}{c}\text { Monarch } \\
{[512 \times 768]}\end{array}$} & $\mathrm{PSNR} / \mathrm{dB}$ & 29.43 & 30.76 & 30.27 & 31.49 & 31.00 & 31.87 \\
\hline & SSIM & 0.919 & 0.935 & 0.925 & 0.939 & 0.936 & 0.943 \\
\hline & Time/s & 0.08 & 586.23 & 64.45 & 1117.58 & 50.14 & 2.87 \\
\hline \multirow{3}{*}{$\begin{array}{c}\text { Pepper } \\
{[512 \times 512]}\end{array}$} & $\mathrm{PSNR} / \mathrm{dB}$ & 32.39 & 33.29 & 33.03 & 34.00 & 34.02 & 34.11 \\
\hline & SSIM & 0.870 & 0.878 & 0.874 & 0.885 & 0.885 & 0.887 \\
\hline & Time/s & 0.05 & 391.92 & 42.45 & 742.14 & 33.89 & 1.92 \\
\hline \multirow{3}{*}{$\begin{array}{c}\text { Ppt3 } \\
{[656 \times 529]}\end{array}$} & $\mathrm{PSNR} / \mathrm{dB}$ & 27.31 & 24.65 & 24.66 & 25.37 & 25.12 & 25.48 \\
\hline & SSIM & 0.874 & 0.891 & 0.886 & 0.913 & 0.902 & 0.914 \\
\hline & Time/s & 0.09 & 453.03 & 49.98 & 798.99 & 43.19 & 2.55 \\
\hline \multirow{3}{*}{$\begin{array}{c}\text { Zebra } \\
{[391 \times 586]}\end{array}$} & $\mathrm{PSNR} / \mathrm{dB}$ & 26.63 & 28.02 & 27.43 & 28.83 & 28.45 & 28.58 \\
\hline & SSIM & 0.794 & 0.837 & 0.829 & 0.850 & 0.846 & 0.849 \\
\hline & Time/s & 0.05 & 350.36 & 36.59 & 649.20 & 29.20 & 1.80 \\
\hline
\end{tabular}


Continued

\begin{tabular}{ccccccccc}
\hline Image & Parameter & Bicubic & ScSR & Fu et al. He et al. Yeganli & Our \\
\hline \multirow{2}{*}{ Comic } & PSNR/dB & 23.12 & 23.93 & 23.75 & 24.19 & 24.04 & 24.06 \\
{$[361 \times 250]$} & SSIM & 0.699 & 0.757 & 0.747 & 0.768 & 0.762 & 0.762 \\
& Time/s & 0.03 & 115.22 & 13.56 & 240.58 & 15.27 & 0.72 \\
\hline \multirow{4}{*}{ Average } & PSNR/dB & 27.09 & 28.04 & 27.77 & 28.47 & 28.35 & 28.51 \\
& SSIM & 0.803 & 0.832 & 0.826 & 0.841 & 0.838 & 0.842 \\
& Time/s & 0.05 & 324.77 & 35.75 & 610.12 & 29.76 & 1.76 \\
\hline
\end{tabular}

To further verify the effect of the MRDL model, we visualize HR dictionaries learned by three methods respectively in Fig. 8. As shown in Fig. 8, the HR dictionary of the ScSR method is only on a single resolution and could not express all forms of structural primitives clearly. Fu et al.'s HR dictionaries are trained by unclassified training patches, that is, all training images are divided into $5 \times 5$ patches to train the dictionary without considering the image content.

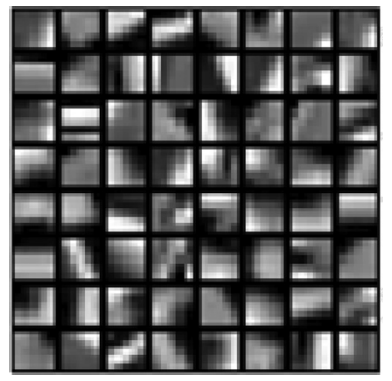

(a) Trained by ScSR method with patch size $5 \times 5$

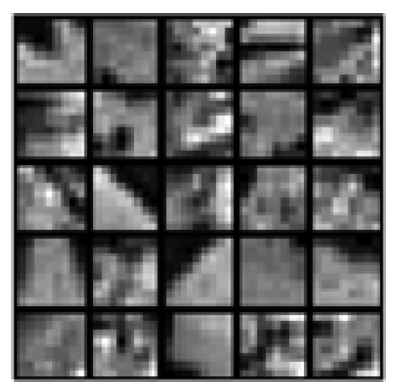

(c) Trained by Fu et al.'s method with patch size $8 \times 8$

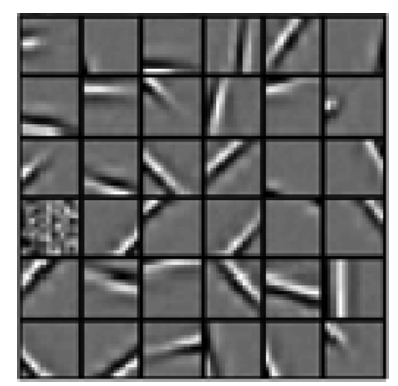

(e) The second resolution dictionary trained by our method with patch size $4 \times 4$

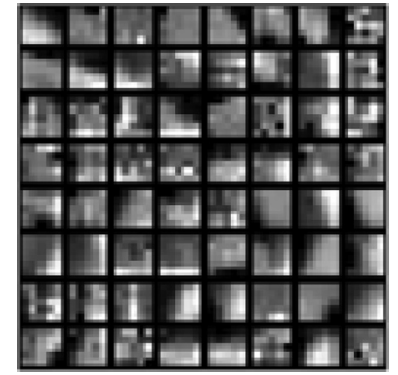

(b) Trained by Fu et al.'s method with patch size $5 \times 5$

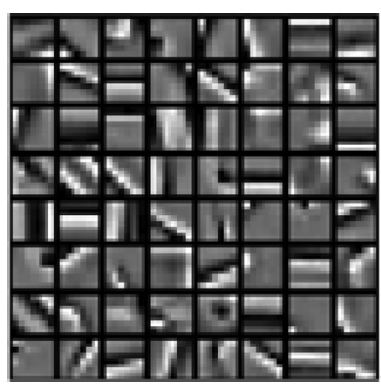

(d) The third resolution dictionary trained by our method with patch size $2 \times 2$

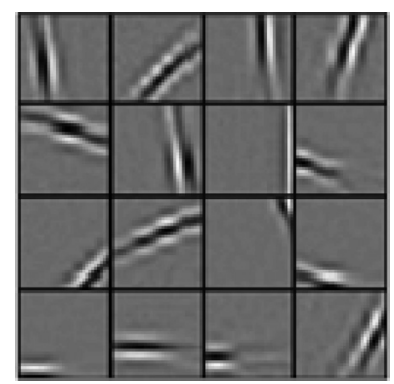

(f) The first resolution dictionary trained by our method with patch size $8 \times 8$

The dictionary with patch size $8 \times 8$ is obtained by the similar way. Thus, as illustrated in Fig. 8, their dictionaries could not completely express the edges and details yet. In our MRDL model, each resolution dictionary is trained by the corresponding category of feature patches, which are extracted from special areas in training images. The visualization results demonstrate that our multi-resolution dictionaries acquire images edges of multiple resolutions in all orientations, which are more expressive and better at recovering image edges.

\section{Conclusions}

This paper presented a fast single image SR approach based on sparse representation and multi-resolution dictionary learning (MRDL). Considering the fact that most natural images simultaneously have flat areas and detailrich areas, and the contents vary significantly across different areas, our proposed adaptive patch partition method (APPM) partitions different areas of images into different size patches. Trained by different size patches rather than fixed-size patches, the novel multi-resolution dictionaries are obtained to express nature images through structural primitives of multiple resolutions. Based on MRDL, our SR algorithm performs well on both recovering image edges and diminishing reconstruction artifacts. Moreover, the reconstruction time is significantly reduced, due to the patch partition and adaptive dictionary size. The experiment results on natural images demonstrate that our proposed method is competitive or even superior to other similar SR methods in terms of PSNR and visual perception, but far superior in terms of computational efficiency.

\section{References}

[1] ZHU Z, GUO F, YU H, et al. Fast single image superresolution via self-example learning and sparse representation. IEEE Trans. on Multimedia, 2014, 16(8): 2178-2190.

[2] FU C H, CHEN H, ZHANG H, et al. Single image super resolution based on sparse representation and adaptive dictionary selection. Proc. of the 19th International Conference on Digital Signal Processing, 2014: 449-453.

[3] VILLENA S, VEGA M, BABACAN S D, et al. Bayesian combination of sparse and non-sparse priors in image super resolution. Digital Signal Processing, 2013, 23(2): 530-541.

[4] HE L, QI H, ZARETZKI R. Beta process joint dictionary learning for coupled feature spaces with application to single image super-resolution. Proc. of the IEEE Computer Society Conference on Computer Vision and Pattern Recognition, 2013: 345-352.

[5] ZEYDE R, ELAD M, PROTTER M. On single image scale-up using sparse-representations. Proc. of the International Conference on Curves and Surfaces, 2012: 711-730.

[6] WANG S L, ZHANG L, LIANG Y, et al. Semi-coupled dictionary learning with applications to image super-resolution and photo-sketch synthesis. Proc. of the IEEE Conference on Computer Vision and Pattern Recognition, 2012: 2216-2223.

Fig. 8 Illustration of HR dictionaries trained by three methods 
[7] DONG W, ZHANG L, SHI G, et al. Image deblurring and super-resolution by adaptive sparse domain selection and adaptive regularization. IEEE Trans. on Image Processing, 2011, 20(7): $1838-1857$.

[8] BABACAN S D, MOLINA R, KATSAGGELOS A K. Variational Bayesian super resolution. IEEE Trans. on Image Processing, 2011, 20(4): 984-999.

[9] YANG J, WRIGHT J, HUANG T, et al. Image super-resolution via sparse representation. IEEE Trans. on Image Processing, 2010, 19(11): $2861-2873$.

[10] YANG J, WRIGHT J, HUANG T, et al. Image super-resolution as sparse representation of raw image patches. Proc. of the IEEE Conference on Computer Vision and Pattern Recognition, 2008: 1-8.

[11] HE Y, YAP K H, CHEN L, et al. A nonlinear least square technique for simultaneous image registration and superresolution. IEEE Trans. on Image Processing, 2007, 16(11): $2830-2841$.

[12] DAI S, HAN M, XU W, et al. Soft edge smoothness prior for alpha channel super resolution. Proc. of IEEE Computer Society Conference on Computer Vision and Pattern Recognition, 2007: $1-8$.

[13] LI X, ORCHARD M T. New edge-directed interpolation. IEEE Trans. on Image Processing, 2001, 10(10): 1521 - 1527.

[14] UR H, GROSS D. Improved resolution from subpixel shifted pictures. Cvgip Graphical Models \& Image Processing, 1992, 54(2): $181-186$.

[15] KEYS R G. Cubic convolution interpolation for digital image processing. IEEE Trans. on Acoustics Speech \& Signal Processing, 1982, 29(6): $1153-1160$.

[16] YANG C Y, YANG M H. Fast direct super-resolution by simple functions. Proc. of the IEEE International Conference on Computer Vision, 2013: 561-568.

[17] YANG J, LIN Z, COHEN S. Fast image super-resolution based on in-place example regression. Proc. of the IEEE Conference on Computer Vision \& Pattern Recognition, 2013: 10591066.

[18] YU J, GAO X, TAO D, et al. A unified learning framework for single image super-resolution. IEEE Trans. on Neural Networks \& Learning Systems, 2014, 25(4): 780-792.

[19] ZHU Y, ZHANG Y, YUILLE A L. Single image superresolution using deformable patches. Proc. of the IEEE Conference on Computer Vision and Pattern Recognition, 2014: $2917-2924$.

[20] YEGANLI F, NAZZAL M, OZKARAMANLI H. Image super-resolution via sparse representation over multiple learned dictionaries based on edge sharpness and gradient phase angle. Signal Image \& Video Processing, 2015, 9(1): $285-293$.

[21] TRINH D H, LUONG M, DIBOS F, et al. Novel examplebased method for super-resolution and denoising of medical images. IEEE Trans. on Image Processing, 2014, 23(4): 1882 1895.

[22] LIN D, TANG X. Coupled space learning of image style transformation. Proc. of the IEEE International Conference on Computer Vision, 2005, 2: 1699-1706.

[23] KANUNGO T, MOUNT D M, NETANYAHU N S, et al. An efficient k-means clustering algorithm: analysis and implementation. IEEE Trans. on Pattern Analysis \& Machine Intelligence, 2002, 24(7): $881-892$.

[24] LEE H, BATTLE A, RAINA R, et al. Efficient sparse coding algorithms. Proc. of the Neural Information Processing Systems, 2007: $721-728$.
[25] MOORE B. Principal component analysis in linear systems: controllability, observability, and model reduction. IEEE Trans. on Automatic Control, 1981, 26(1): 17-32.

[26] ZHOU W, CONRAD B A, RAHIM S H, et al. Image quality assessment: from error visibility to structural similarity. IEEE Trans. on Image Processing, 2004, 13(4): 600-612.

\section{Biographies}

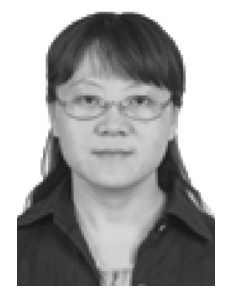

ZHAO Wei was born in 1972. She received her B.S., M.S. and Ph.D. degrees, all from School of Automatic Control of Northwestern Polytechnical University, Xi' an, China. Then she did postdoctoral research in Beihang University, and now she is an associate professor there. Her main research interests are digital image processing, automatic target recognition, signal processing in wireless sensor network and information fusion.

E-mail: zhaowei203@buaa.edu.cn

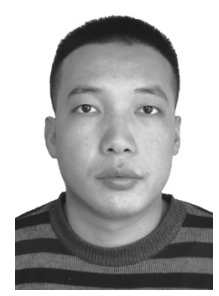

BIAN Xiaofeng was born in 1993. He received his B.E. degree in Beihang University, China, in 2011. $\mathrm{He}$ is currently a master candidate in School of Electronic and Information Engineering of Beihang University, China. His research interest is image processing.

E-mail: xiaofengb@buaa.edu.cn

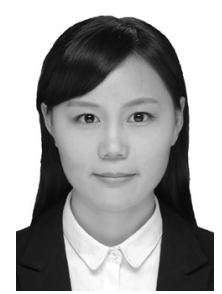

HUANG Fang was born in 1991. She received her B.E. degree in Dalian University of Technology, China, in 2010. She is currently a master candidate in School of Electronic and Information Engineering of Beihang University, China. Her research interest is image processing.

E-mail: huangfang@buaa.edu.cn

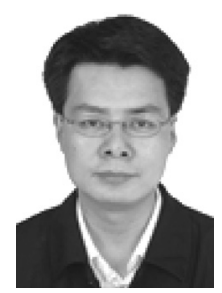

WANG Jun was born in 1972. He received his B.S. degree from Northwestern Polytechnical University, Xi'an, China, in 1995, and M.S. and Ph.D. degrees from Beihang University, Beijing, China, in 1998 and 2001, respectively. He is currently a professor in School of Electronic and Information Engineering, Beihang University. He is interested in signal processing, DSP/FPGA real-time architecture, target recognition and tracking, and so on.

E-mail:wj203@buaa.edu.cn

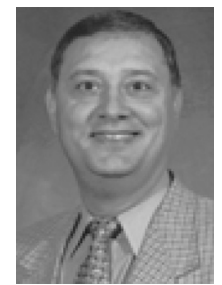

ABIDI Mongi A. was born in 1955. He holds the life-time Cooke-Eversole professorship in the College of Engineering at the University of Tennessee where he served as a faculty since 1987 . He graduated over 75 Masters and Ph.D. students and managed over $\$ 30$ million of external funding in the areas of image processing and robotics. He has published over 320 papers and is co-author or co-editor of four books. His main research interests are data fusion in robotics and machine intelligence, 3D imaging, face identification, and color image processing and applications.

E-mail: abidi@utk.edu 